\title{
Hospital parenteral nutrition-line related sepsis is difficult to define
}

\author{
D. McWhirter, L. Ferguson and R. F. McKee \\ Lister Department of Surgery, Glasgow Royal Infirmary, 84 Castle Street, Glasgow G4 OSF, UK
}

Glasgow Royal Infirmary is a large, tertiary referral teaching hospital, with a multi-disciplinary nutrition team. There are strict guidelines for ward care of our dedicated parenteral nutrition (PN) lines. Hospital policy dictates that if a patient on $\mathrm{PN}$ has a pyrexia of $38^{\circ} \mathrm{C}$ or more, peripheral and central line blood cultures (BC) are taken and the feed stopped unless there is another clear source. The line tip should be sent for culture when the line is removed. An ongoing database record is kept of line insertions and complications. Contemporaneous records of the clinical impression of whether there has been line sepsis have been kept since 1998.

These records were reviewed for 2002-2006 together with the microbiology database to obtain an audit of PN related sepsis. 'Clinical PN line sepsis' was defined as pyrexia associated with PN which settles if the PN line is not used and where there is no other proven source of sepsis.

\begin{tabular}{ccccc}
\hline Year & Line d & $\begin{array}{c}\text { Clinical PN } \\
\text { line sepsis }\end{array}$ & $\begin{array}{c}\text { Clinical PN line sepsis }+ \\
\text { positive BC }(\mathrm{n} / 1000 \mathrm{~d})\end{array}$ & $\begin{array}{c}\text { Clinical PN line sepsis + positive } \\
\text { BC + positive tip (n/1000d) }\end{array}$ \\
\hline 2002 & 1474 & 7 & $5(3.39)$ & $1(0.68)$ \\
2003 & 1757 & 7 & $7(3.98)$ & $3(1.71)$ \\
2004 & 1060 & 7 & $7(6.60)$ & $3(2.83)$ \\
2005 & 1490 & 5 & $5(3.36)$ & $1(0.67)$ \\
2006 & 1931 & 8 & $7(3.63)$ & $3(1.55)$ \\
\hline
\end{tabular}

The 2006 results were examined in more detail. There were sixty lines inserted during this time. The mean time to infection was $26 \mathrm{~d}$. Of eight lines removed because of clinical suspicion of PN line sepsis, six line tips were sent for culture but only five were analysable as there was a 48-h delay in receipt of one specimen. Four of these five line tips grew significant amounts of bacteria (two C. albicans; one S. aureus; one MRSA). Of seven other line tips sent for culture with no clinical suspicion of infection, coagulase-negative staphylococci were grown in four cases and C. parapsilosis in one case.

We were disappointed to find that only $75 \%$ of clinically suspicious line tips had been sent for culture. Despite the policy of sending all PN tips for culture on removal, only $25 \%$ of all line tips had been sent in 2006. Figures for 2002-2005 were similar.

It would appear that our hospital PN infection rates are reasonable in comparison to the published literature, which quotes a sepsis rate of between 1.3 and 7.8 per 1000 catheter days. However it is clear that infection rates can be expressed in many different ways, and when reading the literature care must be taken to compare like results. It would seem logical that the most accurate results are probably obtained when clinical impression is combined with culture of the peripheral and central blood as well as the line tip. However it is possible that this underestimates infection rates if there are delays in receipt of the line tip by the laboratory. 